\title{
Resident Investigators Excel
}

\author{
DC Covey*
}

Department of Orthopaedic Surgery University of California, USA

*Corresponding author: DC Covey, Department of Orthopaedic Surgery University of California, San Diego, USA

\section{Introduction}

On September 5, 2018, the fifth iteration of the Orthopaedic Research and Education Foundation (OREF)/Orthopaedic Research Society (ORS) Southwest Region Resident Research Symposium was held in San Diego, California. The event showcased resident researchers from University of California, San Diego, Stanford University, University of California, San Francisco, Naval Medical Center San Diego, Cedars-Sinai Medical Center, the San Francisco Orthopaedic Residency Program, the University of California, Davis the University of Arizona and Loma Linda University. Papers were judged by clinical and basic science faculty members from the University of California, San Diego; Naval Medical Center San Diego; and by OREF Visiting Professor, Nicholas M. Bernthal MD, Assistant Professor in Residence, Department of Orthopaedic Surgery, University of California, Los Angeles. Hosted by Reid A. Abrams, MD, Interim Chairman and Professor, Department of Orthopaedic Surgery, University of California, San Diego, the OREF ORS Southwest Region Resident Research Symposium has continued to grow since its inception in 2009. This year, 26 residents were selected to present at the symposium. "We were impressed by the number of excellent abstracts. The winners in both the clinical and basic science categories presented impressive, high-quality research," according to D.C. Covey, MD, the local event coordinator.

Participating residents found the event both rewarding and thought-provoking. According to Stephanie Wong, MD, "The OREF has long been dedicated to those in orthopaedics early in their careers which is underscored by the Resident Research Symposiums. The 2018 Southwest Resident Research Symposium was a well-organized conference exhibiting excellent research with renowned faculty speakers and judges. The OREF award is an important recognition of resident research. I am grateful to the OREF for its support of my research and for advancing musculoskeletal research and innovation." Dylan Kluck, MD added that, "Having the opportunity to present this research in front of a regional conference and field questions from an impressive guest speaker was a truly inspiring experience and highlighted the role that we, even as residents, can have in advancing the orthopedic field. In particular, winning the $2^{\text {nd }}$ place Clinical Science OREF award was a true honor and one of the highlights of my career thus far." Trevor Shelton, MD, stated that, "Winning one of the research awards meant a lot as far as validating the time invested in this project and other research projects to hopefully bring forth clinically useful research." "This award helps to advance my goal of becoming a surgeon-scientist studying novel ways to prevent muscle degeneration and fatty infiltration following rotator cuff tears," according to Michael Davies, MD.

Congratulations to the winners!

The judges named the following winners, each of whom received an honorarium:

\section{Basic Science}

$\mathbf{1}^{\text {st }}$ Place: Michael Davies MD, University of California, San Francisco. Altering Stem Cell Fate to Decrease Muscle Degeneration After Rotator Cuff Tears. Study significance:

Our group used a mouse model of rotator cuff tear to replicate the pathology seen after cuff tears in humans and to study the muscle stem cell populations that play a role in the pathogenesis of fatty infiltration. Our recent work suggests that adipogenic differentiation of fibro-adipogenic progenitors present in muscle can be inhibited by histone deacetylase inhibitors, leading to less fat both in vitro and in vivo using our injury model.

$2^{\text {nd }}$ Place: Mario Vargas-Vila MD PhD, University of California, San Diego. Transcriptional Profile of Supraspinatus Muscle in Rabbit Model of Rotator Cuff Tear Over Time. Study significance:

Our study aimed to better characterize muscle degeneration in rotator cuff disease. We used a rabbit model to do this at both the cellular and molecular level. 
$3^{\text {rd }}$ Place: Derek Ju MD, Cedars-Sinai Medical Center, Electrospun Synthetic Bone Scaffold Promotes Mesenchymal Stem Cell Function and rhBMP-2 Mediated Spinal Fusion. Study significance:

My research focuses on osteoinductive synthetic bone scaffolds for spinal fusion.

\section{Clinical Science}

$\mathbf{1}^{\text {st }}$ Place: Stephanie Wong MD, University of California, San Francisco. Effect of Gender on Outcomes After Reverse Total Shoulder Arthroplasty. Study significance:

In this study, we showed that males achieve significantly higher SF-12 PCS and ASES function scores postoperatively compared to females. This may be attributed to differences in ADLs, especially those that require shoulder rotation. All patients experienced improvements in forward elevation and abduction, decrease in pain, and improvement in function. However, patients should be aware that shoulder arthroplasty alone may not change their shoulder rotation. Addressing gender differences in outcomes after surgery will further our understanding of how musculoskeletal conditions affect males and females differently and would help optimize evaluation and treatment of orthopaedic conditions.

$2^{\text {nd }}$ Place: Dylan Kluck MD, University of California, San Diego. A Novel 3D Parameter Can Guide Concave Rod Contour for the
Correction of Lost Kyphosis in Adolescent Idiopathic Scoliosis. Study significance:

"As part of my UCSD research year I was fortunate enough to work in Dr. Peter Newton's lab at Rady Children's Hospital where I was involved in several projects centering primarily around adolescent idiopathic scoliosis. As part of his lab I worked on a longstanding project analyzing intra-op rod contours during posterior spinal fusion to quantify the ideal amount of rod contour required to achieve a desired amount of surgical correction. During my research year, our biomechanical engineer was instrumental in identifying a new 3D parameter which we found to strongly correlate with restoration of thoracic kyphosis. Using 3D spinal reconstructions, this parameter represents an exciting step in being able to estimate a starting point for ideal rod contour.

$3^{\text {rd }}$ Place: Trevor Shelton MD, University of California, Davis. ACL Graft with Hamstring Tendons: Optimal Location for PreOperative MRI Measurement of Cross Sectional Area. Study significance:

The goal of my research project was to determine where along the hamstring tendons had the best correlation to intraoperative graft size for autograft hamstring ACL reconstruction. Using this, we created a model to predict whether hamstring autograft would be of sufficient size for ACL reconstruction.

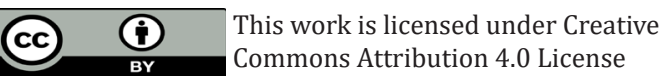

To Submit Your Article Click Here: Submit Article

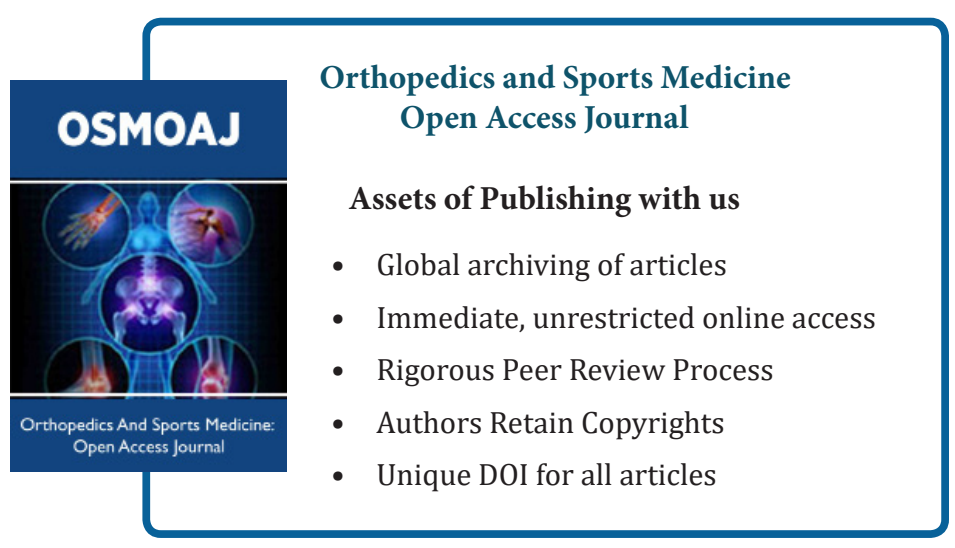

